Title : will be set by the publisher

Editors : will be set by the publisher

EAS Publications Series, Vol. ?, 2007

\title{
WHITE DWARFS AS ASTROPARTICLE PHYSICS LABORATORIES
}

\author{
Jordi Isern ${ }^{1}$ and Enrique García-Berro ${ }^{2}$
}

\begin{abstract}
White dwarfs are well studied objects. The relative simplicity of their physics allows to obtain very detailed models which can be ultimately compared with their observed properties. Among them there is a specific class of stars, the ZZ-Ceti stars that are characterized by the extreme stability of their periods of pulsation. The rate of change of the period is closely related to the characteristic cooling time of the star, which can be accurately computed. This property not only allows to directly test the evolution of white dwarfs but also to constrain parameters of any new physical theory able to perturb the cooling regime. This technique has been successfuly aplied to the case of axions and can be used to constrain the properties of other theoretical particles. The program we propose here consists in using the ability of the Antartic plateau to perform long time and non-interrupted observations to establish the seismological properties of a well defined set of variable white dwarfs (and other stable pulsators).
\end{abstract}

\section{Introduction}

Astrophysical arguments and observations have become a routine tool to obtain empirical information or constraints on existing or hypothetical elementary particles. One of the most important reasons for this is that the dense environment of stars is potentially a powerful source of low-mass weakly interacting particles. Since these particles subsequently escape from the star, this mechanism constitutes a sink of energy that ultimately modifies the stellar evolution. This is particularly useful since, as it is well known, the different non-standard theories leave open the possibility that several exotic particles could exist.

\footnotetext{
${ }^{1}$ Institut de Ciències de l'Espai (CSIC - IEEC), Campus UAB, Torre C5-parells $2^{\text {na }}, 08193$ Bellaterra, Spain

2 Departament de Física Aplicada, Escola Politècnica Superior Castelldefels, Universitat Politècnica de Catalunya, Avda. del Canal Olímpic s/n, 08860 Castelldefels \& Institut d'Estudis Espacials de Catalunya
} 
White dwarfs represent the last evolutionary stage of stars with masses in the range of 8 to $\sim 11 M_{\odot}$. Their structure is rather simple since they have a core that contains the bulk of mass - made of a mixture of carbon and oxygen (for core masses $M \leq 1.05 M_{\odot}$ ) or oxygen and neon (for core masses $M \geq 1.05 M_{\odot}$ ) - which is surrounded by a buffer made of pure helium $\left(M_{\mathrm{He}}<10^{-2} M_{\odot}\right)$ and a tiny envelope made of pure hydrogen $\left(M_{\mathrm{H}}<10^{-4} M_{\odot}\right)$ that control the outwards flux of energy. In some cases the $\mathrm{H}$ envelope is not present. White dwarfs with hydrogen atmospheres are know as DA, if this hydrogen atmosphere is missing they are classified as DO, DB, DC,... depending on their spectral characteristics. Since the core is completely degenerate the star cannot obtain energy from nuclear reactions and its evolution is just a gravothermal process of contraction and cooling that can be roughly described as:

$$
L_{0}=-\frac{d(E+\Omega)}{d t}
$$

where $E$ is the total internal energy and $\Omega$ is the total gravitational energy.

ZZ-Ceti stars are DA white dwarfs that experience $g$-mode pulsations triggered by the $\kappa$-mechanism. The rate of cooling and the rate of change of the period of pulsation of these white dwarfs are related by

$$
\frac{d \ln P}{d t} \propto-\frac{d \ln T}{d t}
$$

(Isern et al. 1992, 1993). Therefore, the observed rate of change of the pulsation period $\dot{P}_{\text {obs }}$ and the rate of change of the period given by the models $\dot{P}_{\bmod }$ when an extra emission is included obeys the following expression:

$$
\frac{L_{0}+L_{1}}{L_{0}}=\frac{\dot{P}_{\mathrm{obs}}}{\dot{P}_{\mathrm{mod}}}
$$

which allows to bound the effects of the extra terms if both $\dot{P}_{\text {obs }}$ and $\dot{P}_{\text {mod }}$ are known. This technique has been successfully applied to bound, for instance, the mass of the axion, the magnetic momentum of neutrinos, the secular variation of the constant of gravitation as well as the compactification of extra dimensions.

G117-B15A is a typical ZZ-Ceti star whose variability was first discovered by McGraw \& Robinson (1976). Since then on, it has been monitored almost continuously. Its mass and effective temperature have been estimated to be $M_{\star} \approx 0.59 M_{\odot}$ and 11,620 K, respectively (Bergeron et al. 1995). Regarding the variability of this star, its observed periods are 215.2, 271.0 and $304.4 \mathrm{~s}$ together with harmonics and linear combinations of the quoted periods (Kepler et al 1982). Of particular interest is the fact that for the $215.2 \mathrm{~s}$ mode it has been possible to find its rate of change, $\dot{P}$. The first published value of $\dot{P}$ (Kepler et al 1991) was obtained using all the data obtained from 1975 to 1990 and was $\dot{P}=(12.0 \pm 3.5) \times 10^{-15} \mathrm{~s} \mathrm{~s}^{-1}$, much larger than the theoretical predictions. More recently, with a much longer time interval of acquired data, Kepler et al.(2000) determined again $\dot{P}$ finding a significantly lower value of $\dot{P}=(2.3 \pm 1.4) \times 10^{-15} \mathrm{~S} \mathrm{~s}^{-1}$. In order to use these 
data, we have first looked for a model that matches the three observed modes as good as possible. After having such a fiducial model, the computation of the theoretical $\dot{P}$ for different cooling rates induced by exotic physical properties is rather straightforward (Córsico et al. 2001).

Evolutionary an seismological calculations clearly indicate that the mass of G117-B15A should be very close to $0.55 M_{\odot}$ and that the mass of the hydrogen mass envelope should also be close to $M_{\mathrm{H}} / M_{\star}=10^{-4}$. The model that provides the best fit to the observations $\left(M_{\star}=0.55 M_{\odot}, l=1, k=2,3,4\right.$ and $\log M_{\mathrm{H}} / M_{\star}=$ $-4.0)$ will be hereafter referred to as the fiducial model. The rate of change of the period for this model is $\dot{P}=3.9 \times 10^{-15} \mathrm{~S} \mathrm{~s}^{-1}$. The discrepancy between this value and the measured rate of change of the period of the $215.2 \mathrm{~s}$ mode can be attributed to the theoretical uncertainties and to the proper motion and parallax (Pajdosz, 1995; Kepler et al, 2000).

The similitude of the theoretical and the seismologically derived values of $\dot{P}$ indicates that the cooling theory of white dwarfs is essentially correct and suggests that there is no room fo any additional cooling. Unfortunately, the erros bars are still too large and prevent from drawing a definite conclusion on both questions.

\section{The case of axions}

There are two types of axion models the so-called KVSZ model and the DFSZ model. The first one couples to hadrons and photons whereas the second one also couples to charged leptons. The coupling strength depends on the specific implementation of the Peccei-Quinn mechanism through dimensionless coupling constants, that are related to the mass. Both the KVSZ and the DFSZ model do not set any constraint on the mass of the axion which must be obtained from experimental tests. For the case of white dwarfs, the dominant process is electron bremsstrahlung emission of DFSZ axions.

In order to compute the effects of axion emission on the evolutionary time scale of G117-B15A and its effect on the expected value of $\dot{P}$ for the $l=1$, $k=2$ mode. Córsico et al (2001) have run an additional set of cooling sequences with different axion masses (and considered the pulsational characteristics in the relevant effective temperature range) for the fiducial model, starting from the same initial conditions used for the models computed without axion emission. They have found that, even considering a wide range for the mass of the axion, the period of the $l=1, k=2,3$, and 4 modes show a very small variation for the whole considered interval. This is indeed a very fortunate situation that allowed us to identify first the structure of the fiducial model without considering axion emission and then to incorporate the axion emissivity. In sharp contrast with the small variation of the computed values of the periods of all the modes with the axion emissivity it was found that the value of $\dot{P}$ for the three identified modes is extremely sensitive. These calculations allowed to obtain the value of $\dot{P}$ of the $l=1, k=2$ mode of the fiducial model at $T_{\text {eff }}=11,620 \mathrm{~K}$ as a function of the mass of the axion and to fix an upper limit of the axion mass by imposing that the value of $\dot{P}$ should be lower than the observed value plus two times the 
standard deviation, that is, lower than $5.1 \times 10^{-15} \mathrm{~S} \mathrm{~s}^{-1}$. This limit was to be $m_{a} \leq 3.97 \cos ^{2} \beta \mathrm{meV}$ in the case of DFSZ axions.

\section{Conclusions}

It is clear that the stability of the periods of pulsation of ZZ-Ceti stars and the dependence of the secular variation of these periods on the cooling rate of such stars make them excellent laboratories to test new physics beyond standard models. Nevertheless, the necessary condition to achieve this goal is to have a significative set of pulsating white dwarfs with well identified periods and well determined secular variations. The ability to follow the light curves during long times without interruptions makes Antartica one of the best sites for conducting such kind of experiments.

\section{Acknowledgements}

This work has been partially supported by the MEC grants AYA04-094-C03-01/02, by the European Union FEDER funds and by the AGAUR.

\section{References}

Bergeron, P., Wesemael, F., Lamontagne, R., Fontaine, G., Saffer, R.A., Allard, N.F., 1995, ApJ, 449, 258

Córsico, A.H., Benvenuto, O.G., Altahus, L.G., Isern, J., García-Berro, E. 2001, New Astron., 6, 197

Isern, J., Hernanz, M., García-Berro, E., 1992, ApJ, 392, L23

Isern, J., Hernanz, M., García-Berro, E., 1993, in "White Dwarfs: Advances in Observations and Theory", Ed.: M.A. Barstow (Dordrecht: Kluwer Academic Publishers), 139

Kepler, S.O., Robinson, E.L., Nather, R.E., McGraw, J.T., 1982, ApJ, 254, 676

Kepler, S.O., et al., 1991, ApJ, 378, L45

Kepler, S.O., Mukadam, A., Winget, D.E., Nather, R.E., Metcalfe, T.S., Reed, M.D., Kawaler, S.D., Bradley, P.A., 2000, ApJ, 534, L185

McGraw, J.T., Robinson, E.L., 1976, ApJ, 205, L155

Pajdosz, G., 1995, A\&A, 295, L17 